\title{
Hypermethylation of serotonin transporter gene in bipolar disorder detected by epigenome analysis of discordant monozygotic twins
}

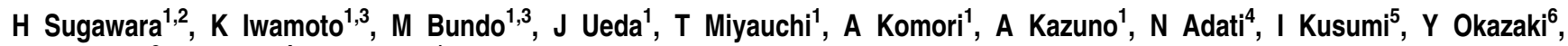 \\ J Ishigooka ${ }^{2}$, T Kojima ${ }^{4}$ and T Kato ${ }^{1}$
}

Bipolar disorder (BD) is a severe mental disorder characterized by recurrent episodes of mania and depression. Serotonin transporter (HTT) is a target of antidepressants and is one of the strongest candidate molecules of mood disorder, however, genetic study showed equivocal results. Here, we performed promoter-wide DNA methylation analysis of lymphoblastoid cell lines (LCLs) derived from two pairs of monozygotic twins discordant for BD. To rule out the possible discordance of copy number variation (CNV) between twins, we performed CNV analysis and found the copy number profiles were nearly identical between the twin pairs except for immunoglobulin-related regions. Among the three genes we obtained as candidate regions showing distinct difference of DNA methylation between one of the two pairs, hypermethylation of SLC6A4, encoding HTT, in the bipolar twin was only confirmed by bisulfite sequencing. Then, promoter hypermethylation of SLC6A4 in LCLs of BD patients was confirmed in a case-control analysis. DNA methylation of SLC6A4 was significantly correlated with its mRNA expression level in individuals with the S/S genotype of HTTLPR, and mRNA expression level was lower in BD patients carrying the S/S genotype. Finally, DNA methylation of the same site was also higher in the postmortem brains of BD patients. This is the first study to report the role of epigenetic modification of SLC6A4 in BD using an unbiased approach, which provides an insight for its pathophysiology. Translational Psychiatry (2011) 1, e24; doi:10.1038/tp.2011.26; published online 26 July 2011

\section{Introduction}

Mood disorders such as major depression and bipolar disorder (BD) cause severe social burdens and there is an urgent need to elucidate the pathophysiology of these disorders. Studying the mechanisms of action of psychotropic drugs has been a major strategy to understand the pathophysiology of mental disorders, and research has focused on serotonin transporter (HTT), the target molecule of antidepressants. Altered density of HTT in the brains of patients with mood disorders has been reported in postmortem samples ${ }^{1}$ and by in vivo molecular imaging. ${ }^{2-6} \mathrm{~A}$ serotonintransporter-linked promoter region (HTTLPR) includes the functional polymorphism, in which the short (S) allele has lower promoter activity than the long (L) allele, ${ }^{7}$ and it has been implicated in mood disorders. ${ }^{1,8-10}$ The $S$ allele was reported to be a risk factor for mood disorders, interacting with stress and/or early environment. ${ }^{8}$ However, the results of subsequent studies are conflicting ${ }^{11-13}$ and none of the recent genome-wide association studies supported the role of SLC6A4 in mood disorders. ${ }^{14}$

We have been searching for gene expression and epigenetic differences between monozygotic (MZ) twins discordant for $\mathrm{BD}$ to elucidate the molecular pathways relevant to its pathophysiology. ${ }^{15,16}$ Although high concordance rate of $\mathrm{BD}$ in $\mathrm{MZ}$ twins supports the contribution of genetic factor in BD, importantly, it is not $100 \%$. Because $M Z$ twins have been regarded as having identical genomes, these facts suggest the importance of environmental or epigenetic factor for the onset of mental disorders. In fact, considerable epigenomic differences between $M Z$ twins have been reported by several groups. ${ }^{17-22}$ On the other hand, there are also reports of genomic differences between $\mathrm{MZ}$ twins such as point mutations, ${ }^{23-25}$ microsatellite repeat length $^{26}$ and copy number variations $(\mathrm{CNVs}){ }^{27}$ Therefore, both genetic and epigenetic factors may have a role in the discordance of the onset of diseases between MZ twins.

Here, we performed promoter-wide DNA methylation analysis of lymphoblastoid cell lines (LCLs) derived from two pairs of $\mathrm{MZ}$ twins discordant for $\mathrm{BD}$. To rule out the possible discordance of CNV between twins, we performed CNV analysis.

\section{Materials and methods}

Samples. We enrolled two pairs of $M Z$ twins discordant with respect to BD (Supplementary Table S1). The twins

\footnotetext{
${ }^{1}$ Laboratory for Molecular Dynamics of Mental Disorders, RIKEN Brain Science Institute, Saitama, Japan; ${ }^{2}$ Department of Psychiatry, Tokyo Women's Medical University, Tokyo, Japan; ${ }^{3}$ Department of Molecular Psychiatry, Graduate School of Medicine, University of Tokyo, Tokyo, Japan; ${ }^{4}$ Research Equipment Center, Hamamatsu University School of Medicine, Shizuoka, Japan; ${ }^{5}$ Department of Psychiatry, Hokkaido University, Sapporo, Japan and ${ }^{6}$ Tokyo Metropolitan Matsuzawa Hospital, Tokyo, Japan

Correspondence: Dr T Kato, Laboratory for Molecular Dynamics of Mental Disorders, RIKEN Brain Science Institute, 2-1 Hirosawa, Wako, Saitama 351-0198, Japan. E-mail: kato@brain.riken.jp

Keywords: bipolar disorder; DNA methylation; monozygotic twins; serotonin transporter

Received 17 June 2011; accepted 21 July 2011
} 
were 49-year-old males (pair 1, previously reported in Kusumi et al. ${ }^{28}$ ) and 42-year-old males (pair 2). Detailed clinical information was described in previous reports and their zygosities were determined by micro-satellite markers and single nucleotide polymorphisms. ${ }^{15,16}$ We used their genomic DNA derived from LCLs for all analyses.

In the CNV analysis, we used a female sample (NA15500, Coriell Cell Repository, Camden, NJ, USA) as a reference and an unrelated healthy female sample for quality control of real-time PCR analysis. Methods of CNV analysis are shown in Supplementary Information.

To exclude the genomic regions in which DNA methylation is different between peripheral blood leukocytes (PBLs) and LCLs, we used four sets of PBLs and LCLs from the same individuals who were unrelated healthy Japanese males and females. ${ }^{29}$ For the collection of PBLs, blood was collected into a $7 \mathrm{ml}$ tube containing EDTA. Red blood cells were lysed by osmotic shock by adding $40 \mathrm{ml}$ of red blood cell lysis buffer containing $10 \mathrm{~mm} \mathrm{Tris} / \mathrm{HCl}, \mathrm{pH} 7.6,6 \mathrm{mM} \mathrm{MgCl}_{2}$ and $10 \mathrm{~mm} \mathrm{NaCl}$. After centrifugation, the pellet was used for DNA extraction.

For the case-control analysis, we examined 20 unrelated BD patients (12 males and 8 females, $38.8 \pm 13.1$ years old (mean \pm s.d.)) and 20 unrelated control subjects (16 males and 4 females, $38.5 \pm 8.9$ years old) using their genomic DNA derived from LCLs. They were subjects from our previous report, $^{30}$ and all of them were Japanese. Written informed consent was obtained from all the subjects.

Postmortem prefrontal cortices (BA10) derived from BD patients $(N=35)$ and controls $(N=35)$ were provided by the Stanley Foundation Brain Collection (The Stanley Medical Research Institute, Chevy Chase, MD, USA). We were able to use $31 \mathrm{BD}$ samples and 32 control samples for DNA methylation analysis.

This study was approved by the Research Ethics Committee of RIKEN.

Cell cultures. LCLs were previously established by transforming the lymphocytes with Epstein-Barr (EB) virus following standard protocol. ${ }^{31}$ Briefly, lymphocytes were separated from peripheral blood and cultured with RPMI 1640 medium containing $20 \%$ fetal bovine serum (Gibco, Carlsbad, CA, USA), penicillin and streptomycin $\left(50 \mu \mathrm{g} \mathrm{ml}^{-1}\right.$ each) and supernatant of the B95-8 cell culture infected by the EB virus. After the cells showed stable growth, they were passaged two or three times a week using similar medium except for $10 \%$ fetal bovine serum. The cells were then kept frozen and recultured for the experiment. We used low-passage LCLs for case-control studies. Genomic DNA was extracted using Gentra Puregene Cell kit (Qiagen, Hilden, Germany).

\section{DNA methylation analysis}

Enrichment of methylated DNA. We used a MethylCollector Ultra Kit (Active Motif, Carlsbad, CA, USA), which can enrich methylated DNA fragments with as few as five methylated $\mathrm{CpG}$ sites. DNA fragments (100 ng) were sonicated to produce $100-$ to $300-b p$ fragments using a sonicator (Covaris, Woburn, MA, USA) and they were incubated with a His-tagged recombinant MBD2b/MBD3L1 protein complex.
After stringent washing, methylated DNA was eluted and purified using the MiniElute PCR purification kit (Qiagen).

Promoter tiling array. We used a GeneChip Human Promoter 1.0R array (Affymetrix, Santa Clara, CA, USA), which contains 4.6 million probes tiled to cover more than 25500 human promoter regions. Sample preparation for the tiling array was performed according to the Affymetrix chromatin immunoprecipitation assay protocol provided by the manufacturer. Briefly, after amplification, DNA samples were labeled using a GeneChip WT Double-Stranded DNA Terminal Labeling Kit (Affymetrix), and they were then hybridized with Affymetrix GeneChip Human Promoter 1.0R arrays. The arrays were stained and washed using GeneChip Fluidics Station 450 and scanned with the GeneChip 3000 7G Scanner. We used fully CpG unmethylated DNA obtained by whole genome amplification as a reference sample.

Tiling array data analysis. For the detection of methylated regions (MRs), model-based analysis of tiling arrays (MAT) software was used. ${ }^{32}$ By using MAT, the signals of each probe in each array can be standardized individually. Parameters used in the MAT analysis were as follows: bandwidth, 300; maximum gap, 300; minimum number of probes, $10 ; P$ value, $10^{-4}$.

The filtering process was performed with R script (http:// www.r-project.org) using the following steps (Supplementary Figure S4). Scripts are available upon request.

(1) Data of each twin pair were directly compared. The regions showing significant differences between the BD twin and the healthy co-twin were selected. Only those regions containing six or more $\mathrm{CpG}$ sites were further selected because the MethylCollector can collect only the DNA fragments that contain five or more CpG sites. The selected MRs were named BD (bipolar twin)-dominant MRs and $C$ (healthy co-twin)-dominant MRs.

(2) The data of each twin were compared with those of a reference sample (that is, unmethylated DNA). Among the BD-dominant MRs, the regions showing the significantly methylated signal compared with a reference sample in $\mathrm{BD}$ twin but not in healthy co-twin were selected. The selected regions were named BD-specific MRs. C-specific MRs were determined vice versa. This step highlighted the methylation difference between twins and eliminates significant but subtle differences to maximize the chance for successful confirmation by other methods.

(3) The MRs overlapping with a CpG islands were selected. For validation of tiling array data, we arbitrarily selected 13 representative MRs for bisulfite sequencing.

Bisulfite conversion. Either $2 \mu \mathrm{g}$ (for LCL samples) or $500 \mathrm{ng}$ (for postmortem brain samples) of genomic DNA was converted for methylation sequencing using Epitect bisulfite kits (Qiagen), according to the manufacturer's standard protocol. Completion of sodium bisulfite conversion was confirmed during sequencing by ensuring that known lone cytosines were read as thymines. 
Bisulfite sequencing. Primer pairs were determined using Meth Primer software. ${ }^{33}$ PCR products were purified by MinElute PCR Purification Kit (Qiagen) or digested with thermostable $\beta$-agarase (NIPPON GENE, Tokyo, Japan) and cloned using a TOPO TA cloning kit (Invitrogen, Carlsbad, CA, USA). Single bacterial colonies were subjected to sequencing analysis. Each bisulfite sequencing data was quantified by quantification tool for methyaltion analysis. ${ }^{34}$

Filtering of tiling array data. To further narrow the number of MRs by setting more stringent criteria based on the results of bisulfite sequencing, we reset the threshold for filtering as a $P$ value $<10^{-6}$ of direct comparison between each twin pair (Supplementary Figure S5, step 4).

Next, we excluded the regions that showed alteration of DNA methylation status before and after the transformation by EB virus. We previously performed the DNA methylation analysis using the same platform in four sets of PBLs and LCLs established from the same individuals and identified the regions that showed methylation only in LCLs or only in PBLs. ${ }^{30}$ MRs that overlapped with those regions were excluded (Supplementary Figure S5, step 5).

Finally, from the candidate regions for BD-specific MRs, the regions that were detected as MRs in at least one of LCL were excluded (Supplementary Figure S4), because the regions methylated in LCLs of healthy individuals cannot be related to disease. With regard to the C-specific MRs, only the regions overlapping with common MRs in four LCLs were selected. Loss of methylation in patients can be regarded as related to disease only when methylation in that region is consistently seen in healthy individuals (Supplementary Figure S5, step 6).

\section{Case-control analysis}

Pyrosequencing. The PCR product of bisulfite-modified DNA was used for pyrosequencing analysis according to the manufacturer's standard protocol (Qiagen). Briefly, $4 \mu \mathrm{l}$ of streptavidin-sepharose beads (Amersham Biosciences, Piscataway, NJ, USA) and $54 \mu \mathrm{l}$ of binding buffer $(10 \mathrm{~mm}$ Tris- $\mathrm{HCl}, 1 \mathrm{~mm}$ EDTA, $2 \mathrm{M} \mathrm{NaCl}, 0.1 \%$ Tween-20 at $\mathrm{pH} 7.6$ ) were mixed with $50 \mu \mathrm{l}$ of PCR product for $10 \mathrm{~min}$ at room temperature. The reaction mixture was placed onto a MultiScreen-HV, Clear Plate (Millipore, Billerica, MA, USA). After applying the vacuum, the beads were treated with a denaturation solution $(0.2 \mathrm{~N} \mathrm{NaOH})$ for 1 min and washed twice with washing buffer ( $10 \mathrm{~mm}$ Tris-acetate at $\mathrm{pH}$ 7.6). The beads were then suspended with $50 \mu$ of annealing buffer (20 mM Tris-acetate, $2 \mathrm{~mm}$ Mg-acetate at $\mathrm{pH} 7.6$ ) containing $10 \mathrm{pmol}$ of sequencing primer. The template-sequencing primer mixture was transferred onto a PSQ 96 Plate (Biotage, Uppsala, Sweden), heated to $90{ }^{\circ} \mathrm{C}$ for $2 \mathrm{~min}$ and cooled to room temperature. Sequencing reactions were performed with a PyroMark Gold Q96 Reagents Kit (Qiagen) according to the manufacturer's instructions. The percentages of methylation were calculated from the raw data using the allele quantification algorithm of the software provided by the manufacturer (PSQ96MA2.1.1 software, Qiagen). ${ }^{35}$ The PCR primers used for bisulfite sequencing of the promoter region of SLC6A4 were $5^{\prime}$-TTTTTAGTTGTTTG GGTATTTGTGTTA-3' (forward) and 5'-AAAACTTTACAAC CTCTTAAAAACCC-3' (reverse). The sequencing primers were 5'-TTTTGTATAAAGTTATTTGT-3', 5'-AATATAAATTA TGGGTTGAA-3' and 5'-ATTTTTTTTAAGGGGTTTTT-3', and the reading sequences were AT/CGT/CAT, AT/CGA AAGTAAGTAATTITTTTTAAAGT/CG and TAT/CGG.

Genotyping. Using genomic DNA derived from LCLs, we investigated the length polymorphism repeat (HTTLPR) in the promoter region of SLC6A4, which comprises a 44-bp insertion (long, $L$ allele) or deletion (short, $S$ allele), and one single nucleotide polymorphism in $L$ allele (LA/LG), which was reported to be related to gene expression as well as HTTLPR genotype. ${ }^{36}$

For HTTLPR genotype analysis, amplification of genomic DNA was performed as initially described by Kaiser et al., ${ }^{37,38}$ with slight modifications. In a total volume of $10 \mu \mathrm{l}$, we used $40 \mathrm{ng}$ DNA; $200 \mu \mathrm{M}$ each of dATP, dCTP, and dTTP; $150 \mu \mathrm{M}$ dGTP, $50 \mu \mathrm{M}$; 7-deaza-GTP (Roche, Mannheim, Germany); $1 \mu \mathrm{M}$ of each primer; $10^{*}$ buffer 1 ; and 2.8 units of mixed polymerase (Taq- and Tgo-polymerase) from the Expand Long-Range PCR System (Roche). Applied primer sequences were 5'-GCAACCTCCCAGCAACTCCCTGTA-3' (forward) and 5'-GAGGTGCAGGGGGATGCTGGAA-3' (reverse). The PCR program of both reactions consisted of an initial cycle of $2 \mathrm{~min}$ at $94^{\circ} \mathrm{C}$ followed by 35 cycles each consisting of $10 \mathrm{~s}$ at $98^{\circ} \mathrm{C}, 30 \mathrm{~s}$ at $68^{\circ} \mathrm{C}$ and $30 \mathrm{~s}$ at $72^{\circ} \mathrm{C}$. After amplification, HTTLPR amplicons were separated by electrophoresis in $3 \%$ agarose gels after staining with ethidium bromide.

For LA/LG single nucleotide polymorphism analysis, each product of $L$ allele in $L / S$ genotype subjects was digested with thermostable $\beta$-agarase (NIPPON GENE) and subjected to sequencing analysis.

Real-time quantitative RT-PCR. Total RNA was extracted using Trizol (Invitrogen) and cleaned following the standard protocol. After DNase I treatment, $5 \mu \mathrm{g}$ of total RNA was used for complementary DNA synthesis by oligo (dT) 12-18 primer and SuperScript II reverse transcriptase (Invitrogen). Real-time quantitative RT-PCR using Taqman Universal PCR master mix, No AmpErase UNG (Applied Biosystems, a part of Life Technologies, Carlsbad, CA, USA), was performed with an ABI Prism 7900HT (Applied Biosystems). The PCR conditions were denaturation at $95^{\circ} \mathrm{C}$ for $10 \mathrm{~min}$, followed by 40 cycles of $95^{\circ} \mathrm{C}$ for $15 \mathrm{~s}$ and $60^{\circ} \mathrm{C}$ for $60 \mathrm{~s}$. The comparative $\mathrm{Ct}$ method was used to quantify transcripts according to the manufacturer's protocol (User Bulletin 2, Applied Biosystems). Each sample was quantified in triplicate. Amplification of single products was confirmed by monitoring the dissociation curve. We used the expression level of actin beta $(A C T B)$ as an internal control. The standard $\mathrm{ABI}$ primer probe sets were used (SLC6A4, Hs00984356; ACTB, Hs99999903). We performed statistical analysis using SPSS (SPSS Japan, Tokyo, Japan).

\section{Results}

Copy number differences between the twins. The copy number profiles were seemingly identical between both twin pairs (Supplementary Figure S1). To find subtle copy number differences between the twins, we detected their respective 
copy number changes compared with a reference and calculated the fold change of each probe. In both twin pairs, the differences with fold changes of more than 1.2 were mostly found on chromosomes 2, 14 and 22, which contain immunoglobulin (lg)-related regions (Supplementary Figure S2). We examined 40 regions including one Ig-related region by real time PCR analysis. Although the copy number change of Ig-related region was confirmed, none of other regions with copy number differences between the twins were confirmed by real-time PCR analysis (Supplementary Figure S3). This suggested that most of the apparent copy number differences detected by the array were false positives, except for Ig-related regions. The differences of the copy number in Ig-related regions between the twins are reasonable because a single $B$ cell having a specific genome rearrangement of Ig-related gene ${ }^{39}$ is amplified by EB virus transformation in LCLs.

DNA methylation differences between the twins. Promoterwide DNA methylation profiles of the twins were examined by Affymetrix GeneChip Human Promoter 1.0R tilling arrays after methylated DNA was enriched using MBD2b and MBD3L1 conjugated beads. Fully unmethylated DNA obtained by whole genome amplification was used as a reference. Using this method, the DNA methylated status within the $10 \mathrm{~kb}$ range for each of more than 25500 promoters could be analyzed. $\mathrm{MAT}^{32}$ was used to identify MRs. By using MAT, the signals of each probe in each array can be standardized individually.

DNA methylation analysis by the promoter tiling array showed MRs specific to the bipolar twin (80 or 4, respectively) or those to the healthy co-twin (71 or 81 , respectively) in each twin pair (Supplementary Figure S4). Among the 236 regions containing six or more $\mathrm{CpG}$ sites, 13 representative regions overlapping with $\mathrm{CpG}$ islands were analyzed by bisulfite sequencing to validate the tiling array data. Although the DNA methylation differences between the twins were confirmed in most regions, some differences with $P$ values more than $10^{-6}$ could not be confirmed (Supplementary Figures S5 and S6). To narrow the number of candidate MRs, we applied more stringent criteria $\left(P\right.$ value $\leqslant 10^{-6}$ ) based on the results of bisulfite sequencing. We compared the DNA methylation status between four sets of PBLs and LCLs obtained from the same individuals and found that MRs in PBLs were largely conserved in LCLs but a part of the regions were methylated only in LCLs or only in PBLs. ${ }^{30}$ Thus, we eliminated those 'variable' MRs during establishment of LCLs. Furthermore, the regions detected as MRs in at least one of the LCL were excluded from the BD-specific MRs, and only the regions overlapping with common MRs in four LCLs were selected with regard to the C-specific MRs (Supplementary Figure S4). We finally obtained three candidate MRs: KIAA1530 on chromosome 4 as a hypermethylated region in the healthy co-twin, and FANK1 (fibronectin type III and ankyrin repeat domains 1) on chromosome 10 and SLC6A4, encoding serotonin transporter on chromosome 17 (Supplementary Figure S7), as a hypermethylated region in the bipolar twin, only from pair 1.

These three regions were examined by bisulfite sequencing, and we could confirm the differential DNA methylation status between the twins only in the promoter region of SLC6A4 (Figure 1b and Supplementary Figures S8 and S9).

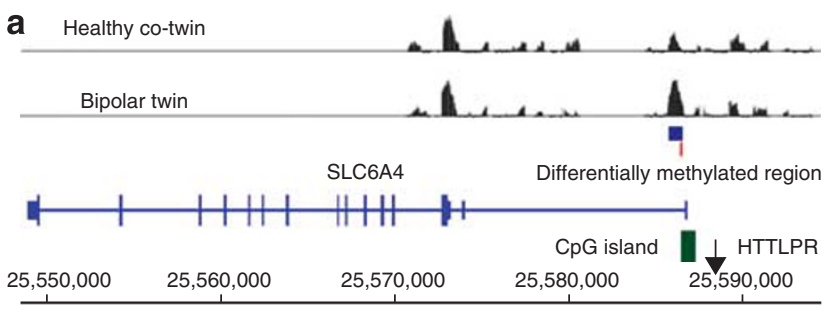

b

AGCCACCTGCACGCGATGACAGCAAAGTAAAGATCAAACATAAACCATGG GTTGAAACGAAAGCAAGCAACTCTTCCCAAAGCGCAGGACAGCACTTTGC TCAAGACCCTCTTTAAGGGGTCTTTCACGGGTCCTCAAGAGGTTGCAAAG
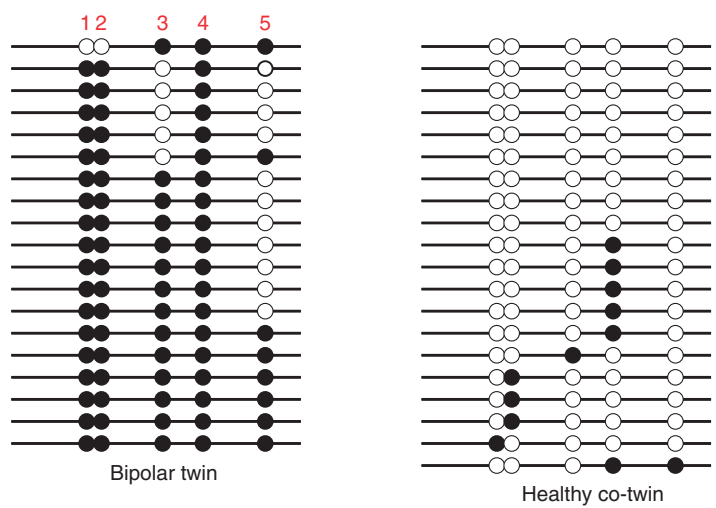

C

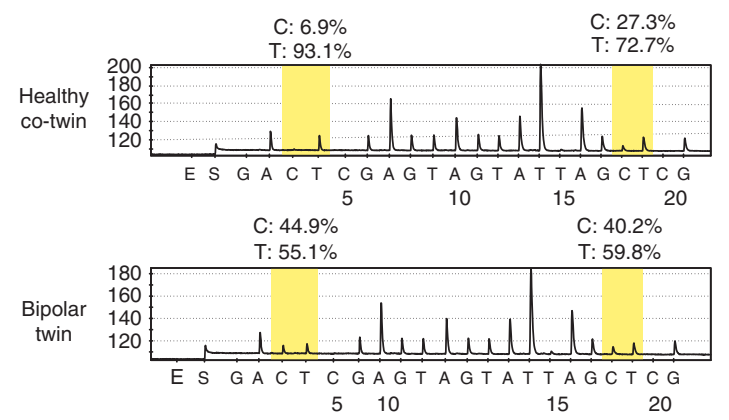

Figure 1 DNA methylation difference between twins in SLC6A4. (a) Results of comprehensive DNA methylation analysis of lymphoblastoid cell lines (LCLs) of a pair of monozygotic twins discordant for bipolar disorder using tiling arrays. The vertical axis represents the signal intensity, and the horizontal axis represents the base number on the chromosome 17 (NCBI36/hg18). Exon-intron structure of the SLC6A4 is shown below the data of tiling arrays. The $\mathrm{CpG}$ island and the regions of HTTLPR are shown by a green square and an arrow, respectively. The region showing statistically significant methylation difference between the bipolar twin and the healthy co-twin, and the region examined by bisulfate sequencing are shown by a blue square and a red bar, respectively. (b) Results of bisulfite sequencing. The genomic region examined by bisulfite sequencing, which corresponds to the base numbers from 25586333 and 25586482 , is shown above. The five $\mathrm{CpG}$ sites are surrounded by red squares. Black and white circles represent the methylated and unmethylated $\mathrm{CpGs}$, respectively. Each raw shows the data of one clone. Five circles in one raw represent the five $\mathrm{CpG}$ sites shown above. This region is methylated in the bipolar twin but not in the healthy co-twin. (c) Representative results of pyrosequencing using independent samples. The results of two $\mathrm{CpG}$ sites ( 3 and 4 ) were shown by yellow shadows. Percentages of $C$ and $T$ mean fractions of methylation and unmethylation on each $\mathrm{CpG}$ site, respectively. Each fraction of methylation on other $\mathrm{CpG}$ sites of the bipolar twin and the healthy cotwin was 24.2 and 0 on CpG1, 28.8 and 0 on CpG2, and 9.3 and 4.3 on CpG5, respectively. The differences of DNA methylation in SLC6A4 between the twins were confirmed using the other LCL samples of the twins recultured for this experiment. 
This region located on the $\mathrm{CpG}$ island shore at the $2-3 \mathrm{~kb}$ downstream of HTTLPR was hypermethylated in the bipolar twin. We also examined the DNA methylation status of the twins using independent cell culture by pyrosequencing to rule out a possibility of cell culture-induced artificial or stochastic change (Figure 1c).

Case-control analysis for DNA methylation of LCLs in SLC6A4. Because there are DNA methylation changes even between healthy $M Z$ twins, the existence of DNA methylation difference does not warrants the relationship with the disease. Thus, we next performed a case-control analysis using the LCLs of 20 patients with BD and 20 controls. The DNA methylation of the two CpG sites (CpG3 and CpG4) was significantly higher in BD patients than in controls $(P<0.05$, Mann-Whitney $U$-test; Figure 2a). We also analyzed the expression level of SLC6A4 in LCLs by real-time quantitative RT-PCR. HTTLPR, including a single nucleotide polymorphism in $L$ allele (LA/LG) that reportedly affects gene expression, was genotyped. ${ }^{36}$ Because all subjects with $L$ allele, but one control subject with $S / L G$ genotype, had LA allele, LA and LG alleles were not separately analyzed. Among the subjects with the S/S genotype, the mRNA expression level was significantly lower $(P<0.05$, Mann-Whitney $U$-test; Figure $2 \mathrm{~b})$ and the DNA methylation level of $\mathrm{CpG} 3$ was significantly higher $(P<0.05$; Mann-Whitney $U$-test; Figure $2 \mathrm{c})$ in the $\mathrm{BD}$ patients compared with controls. The same trend was seen in CpG4 ( $P=0.059$, Mann-Whitney $U$-test). The DNA methylation level of $\mathrm{CpG} 3$ was significantly correlated with its mRNA expression level only in subjects with the S/S genotype ( $r=-0.425, P=0.043, n=23$; Figure $2 d$ ) and not in those with the $\mathrm{S} / \mathrm{L}$ genotype $(r=-0.262, P=0.346$, $n=15$; Figure 2e).

DNA methylation status of postmortem brain samples in SLC6A4. DNA methylation patterns in blood cells may not directly reflect the status of the brain. To test whether hypermethylation of SLC6A4 is also observed in the brain, we examined the DNA methylation status of these
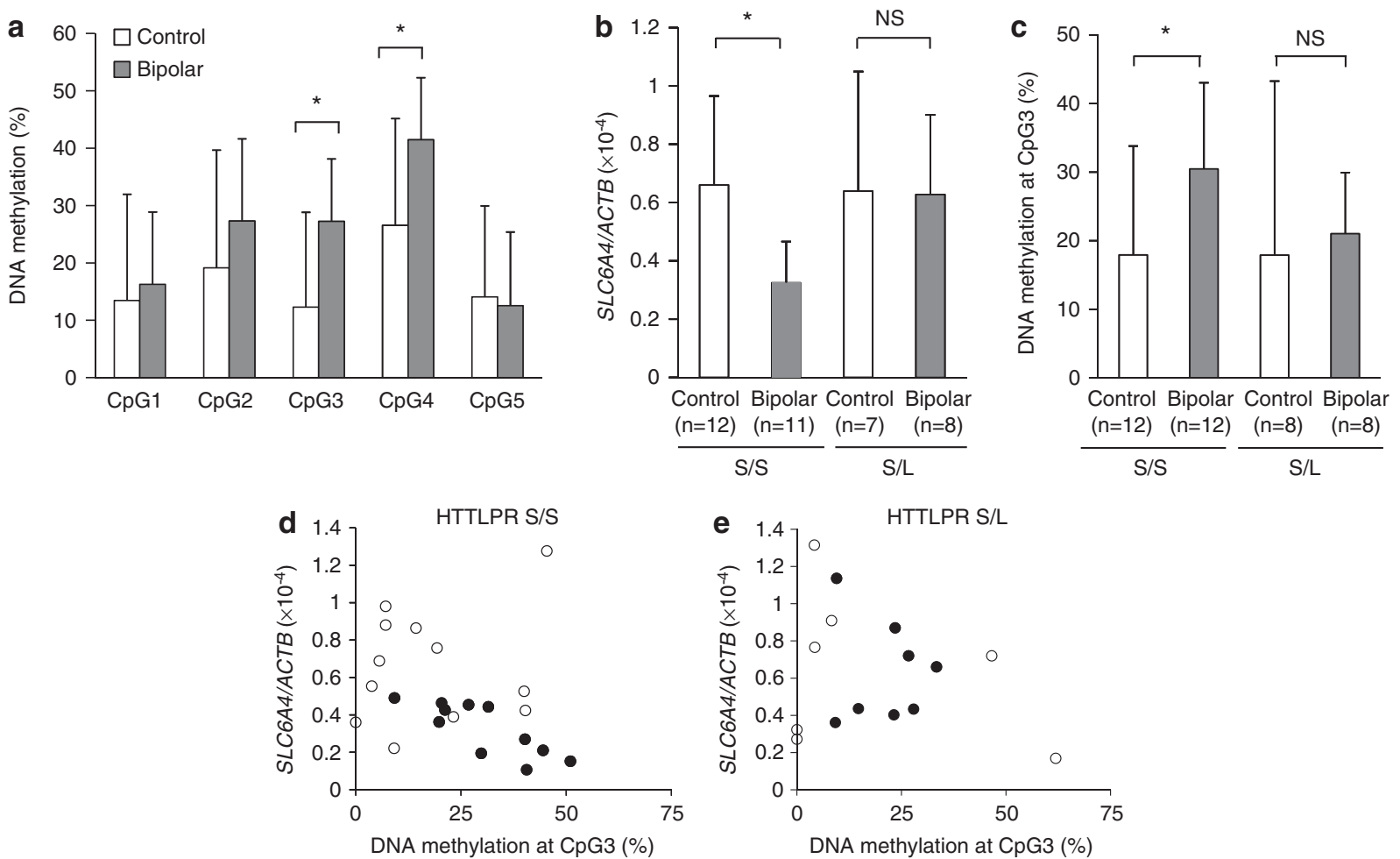

Figure 2 Case-control analysis of DNA methylation and gene expression in LCLs. (a) DNA methylation levels of five CpG sites shown in the Figure 1 in LCLs (bipolar disorder (BD), $n=20$, controls, $n=20$ ) using pyrosequencing. Two $\mathrm{CpG}$ sites (3 and 4) showed significantly higher DNA methylation in BD patients. Values indicate mean \pm s.d. Asterisk indicates significance; $P=0.008$ and $P=0.035$ for $C p G 3$ and $C p G 4$, respectively, by Mann-Whitney $U$ test. (b) Relationship between expression level of SLC6A4 and HTTLPR genotype (BD, $n=19$, controls, $n=19$ ). One datum in each diagnostic group was turned out to be at outlier by Smirnov-Grubbs test and removed from data analysis. Among the all samples $(n=38)$, the mRNA level of SLC6A4 tended to be lower in BD patients compared with controls $(P=0.08$; Mann-Whitney U-test). The mRNA expression level was significantly lower in BD patients compared with controls only in subjects with the $S / S$ genotype $(P=0.007$; Mann-Whitney $U$-test) and not in those with the $S / L$ genotype $(P=0.867$; Mann-Whitney U-test). Values indicate mean \pm s.d. An asterisk indicates significant difference $(P<0.05)$. NS, not statistically significant. (c) Relationship between the DNA methylation of CpG3 and HTTLPR genotype (BD, $n=20$, controls, $n=20)$. The DNA methylation of the CpG3 site was significantly higher in $\mathrm{BD}$ patients compared with controls only in the subjects with the $\mathrm{S} / \mathrm{S}$ genotype $(P=0.020$, Mann-Whitney $U$-test) but not in those with the $\mathrm{S} / \mathrm{L}$ genotype $(P=0.105$, Mann-Whitney U-test). The DNA methylation of the CpG4 site also tended to be higher in BD patients compared with controls only in the subjects with the S/S genotype ( $P=0.052$, Mann-Whitney U-test) and not in those with the $\mathrm{S} / \mathrm{L}$ genotype $(P=0.279$, Mann-Whitney U-test). Values indicate mean \pm s.d. (d, e) Correlation between the DNA methylation at the CpG3 site and mRNA expression level of $S L C 6 A 4$ (BD, $n=19$, controls, $n=19$ ). There was a significant correlation in the subjects with the $\mathrm{S} / \mathrm{S}$ genotype $(r=-0.425, P=0.043, n=23)$; (d), whereas no significant correlation was found in the subjects with the S/L genotype $(r=-0.262, P=0.346, n=15)$; (e). Closed circles represent patients with $\mathrm{BD}$ and open circles represent control subjects. 


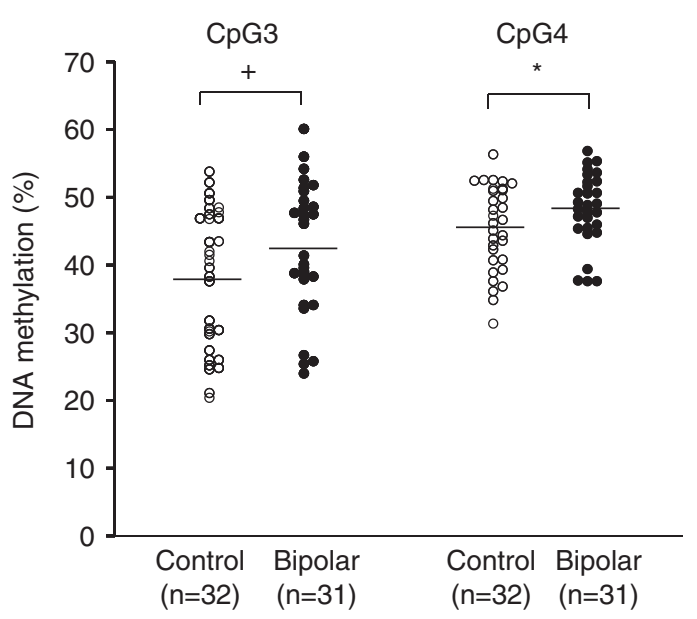

Figure 3 DNA methylation analysis of SLC6A4 in the prefrontal cortices of bipolar disorder (BD) patients and control subjects. The $\mathrm{CpG} 3$ and $\mathrm{CpG} 4$, shown in the Figure 1, were analyzed by pyrosequencing. Closed circles represent patients with $\mathrm{BD}$ and open circles represent control subjects. Horizontal bars indicate averages. ${ }^{*} P<0.05,{ }^{+} P<0.10$ (Mann-Whitney $U$-test, one-tailed). There was no significant difference of gender between the BD patients and controls. Age, postmortem interval (hours) and brain pH were not significantly correlated with the DNA methylation level of both CpG3 and CpG4. In the BD patients, there was no significant difference of the DNA methylation level of the two $\mathrm{CpG}$ sites between the subjects with and without antidepressant treatment.

two $\mathrm{CpG}$ sites in postmortem brains of $31 \mathrm{BD}$ patients and 32 controls obtained from the Stanley Foundation Brain Collection. CpG4 showed a significantly higher DNA methylation level in BD patients compared with controls $(P<0.05$, Mann-Whitney U-test, one-tailed), and CpG3 also showed the same trend $(P=0.059$, Mann-Whitney $U$-test, one-tailed; Figure 3 ). There was no significant effect of confounding factors, such as gender, age, postmortem interval (hours), brain $\mathrm{pH}$ and administration of antidepressants, on the DNA methylation level of the two CpG sites.

\section{Discussion}

Multiple lines of evidence suggest the role of SLC6A4 in BD. Although the results of serotonin transporter-binding potential in BD patients are conflicting, ${ }^{2-6}$ one of them reported the low potential in the depressive state, ${ }^{40}$ and less gene expression of serotonin transporter in postmortem brains of depressed suicide victims was also reported. ${ }^{41}$ HTTLPR was initially reported to be associated with $B D$, though subsequent studies show conflicting results. ${ }^{9,42,43}$ Gene-environment $(G \times E)$ interaction ${ }^{14}$ and resulting epigenetic changes ${ }^{44}$ might explain the inconsistent results of genetic studies in mood disorder. ${ }^{14}$ In this study, we found a possible interaction between HTTLPR genotype and BD diagnosis on the DNA methylation and mRNA expression level of SLC6A4. DNA methylation level of the CpG island upstream of SLC6A4 was reported to be associated with environmental factors ${ }^{45,46}$ and possibly with HTTLPR genotype. ${ }^{47}$ Weaver et al. ${ }^{48}$ hypothesized that DNA methylation plays a role as an epigenetic mark of $\mathrm{G} \times \mathrm{E}$ interaction, and they suggested that hypermethylation of the promoter region of the glucocorticoid receptor gene associated with poor maternal care causes stress vulnerability. Hypermethylation of SLC6A4 in BD may reflect epigenetic effect in $G \times E$ interaction and contribute to the patho physiology of $B D$.

In this study, we performed promoter-wide DNA methylation analysis and detected hypermethylation of SLC6A4 in the bipolar twin. The differences of methylation patterns between $M Z$ twins may depend on age and difference of nurturing environment, ${ }^{18}$ and a twin study showed that the variation of DNA methylation in SLC6A4 is attributable to unique environmental factors rather than heritable factors. ${ }^{49}$ It would be important to elucidate the origin and timing of the methylation change in $S L C 6 A 4^{50}$ in the future study. In this study, only one of the two twins showed marked difference of DNA methylation of SLC6A4. Considering the heterogeneity of BD, it is not realistic to postulate that all patients with $B D$ are caused by the same genetic or epigenetic factor. Thus, a close investigation in a large series of well-characterized discordant twins may provide a clue to understand the pathophysiology of $\mathrm{BD} .{ }^{51}$ In this study, the difference between the groups was relatively modest in case-control studies. There was a large inter-individual difference of DNA methylation at CpG3 in LCLs of patients with BD (9.2 to $51 \%)$, possibly reflecting the heterogeneity of $\mathrm{BD}$. In these analyses, we used genomic DNA derived from LCLs cultured with drug-free medium to minimize the effect of medication on DNA methylation. Although none of BD patients were taking antidepressants at the blood sampling, it is difficult to know what the effects of medications and co-morbid illnesses are on the methylation signatures observed in these samples. Moreover, we only examined a portion of the SLC6A4. Although DNA methylation in LCLs is not the same as PBLs, MRs in PBLs are largely maintained in LCLs. ${ }^{30}$ In this study, we excluded the MRs affected by EB virus transformation and finally detected the hypermethylation of SLC6A4 in the brains of $\mathrm{BD}$ patients. We could only examine the methylation of $S L C 6 A 4$ in prefrontal cortices, but further analysis of other regions expressing high levels of $\mathrm{HTT}$, such as dorsal raphe nucleus, would be interesting.

In summary, we detected hypermethylation of SLC6A4 in $\mathrm{BD}$, using an unbiased approach. DNA methylation of SLC6A4 may be an epigenetic mark resulting from a $\mathrm{G} \times \mathrm{E}$ interaction that leads to the development of BD. Our findings add a new insight to elucidate the pathophysiology of mood disorder.

\section{Conflict of interest}

The authors declare no conflict of interest.

Acknowledgements. Postmortem brain samples were donated by The Stanley Medical Research Institute Brain Collection courtesy of Drs Michael B Knable, E Fuller Torrey, Maree J Webster and Robert H Yolken. We are grateful to Dr Kakiuchi and Ms Ishiwata for the establishment of LCLs of twin pairs, and the Research Resource Center of RIKEN Brain Science Institute for performing the microarray analysis.

1. Mann JJ, Huang YY, Underwood MD, Kassir SA, Oppenheim S, Kelly TM et al. A serotonin transporter gene promoter polymorphism (5-HTTLPR) and prefrontal cortical binding in major depression and suicide. Arch Gen Psychiatry 2000; 57: 729-738.

2. Cannon DM, Ichise M, Fromm SJ, Nugent AC, Rollis D, Gandhi SK et al. Serotonin transporter binding in bipolar disorder assessed using [11C]DASB and positron emission tomography. Biol Psychiatry 2006; 60: 207-217. 
3. Cannon DM, Ichise M, Rollis D, Klaver JM, Gandhi SK, Charney DS et al. Elevated serotonin transporter binding in major depressive disorder assessed using positron emission tomography and [11C]DASB; comparison with bipolar disorder. Biol Psychiatry 2007; 62: 870-877.

4. Chou YH, Wang SJ, Lin CL, Mao WC, Lee SM, Liao MH. Decreased brain serotonin transporter binding in the euthymic state of bipolar I but not bipolar II disorder: a SPECT study. Bipolar Disord 2010; 12: 312-318.

5. Ichimiya T, Suhara T, Sudo Y, Okubo Y, Nakayama K, Nankai M et al. Serotonin transporter binding in patients with mood disorders: a PET study with [11C](+)McN5652. Biol Psychiatry 2002; 51: 715-722.

6. Parsey RV, Hastings RS, Oquendo MA, Hu X, Goldman D, Huang YY et al. Effect of a triallelic functional polymorphism of the serotonin-transporter-linked promoter region on expression of serotonin transporter in the human brain. Am J Psychiatry 2006; 163: 48-51.

7. Heils A, Teufel A, Petri S, Seemann M, Bengel D, Balling U et al. Functional promoter and polyadenylation site mapping of the human serotonin $(5-\mathrm{HT})$ transporter gene. J Neural Transm Gen Sect 1995; 102: 247-254.

8. Caspi A, Sugden K, Moffitt TE, Taylor A, Craig IW, Harrington H et al. Influence of life stress on depression: moderation by a polymorphism in the 5-HTT gene. Science 2003; 301: 386-389.

9. Collier DA, Stober G, Li T, Heils A, Catalano M, Di Bella D et al. A novel functional polymorphism within the promoter of the serotonin transporter gene: possible role in susceptibility to affective disorders. Mol Psychiatry 1996; 1: 453-460.

10. Pezawas L, Meyer-Lindenberg A, Drabant EM, Verchinski BA, Munoz KE, Kolachana BS et al. 5-HTTLPR polymorphism impacts human cingulate-amygdala interactions: a genetic susceptibility mechanism for depression. Nat Neurosci 2005; 8: 828-834.

11. Kendler KS, Kuhn JW, Vittum J, Prescott CA, Riley B. The interaction of stressful life events and a serotonin transporter polymorphism in the prediction of episodes of major depression: a replication. Arch Gen Psychiatry 2005; 62: 529-535.

12. Surtees PG, Wainwright NW, Willis-Owen SA, Luben R, Day NE, Flint J. Social adversity, the serotonin transporter (5-HTTLPR) polymorphism and major depressive disorder. Biol Psychiatry 2006; 59: 224-229.

13. Uher R, McGuffin P. The moderation by the serotonin transporter gene of environmenta adversity in the aetiology of mental illness: review and methodological analysis. Mol Psychiatry 2008; 13: 131-146.

14. Caspi A, Hariri AR, Holmes A, Uher R, Moffitt TE. Genetic sensitivity to the environment: the case of the serotonin transporter gene and its implications for studying complex diseases and traits. Am J Psychiatry 2010; 167: 509-527.

15. Kakiuchi C, Iwamoto K, Ishiwata M, Bundo M, Kasahara T, Kusumi I et al. Impaired feedback regulation of XBP1 as a genetic risk factor for bipolar disorder. Nat Genet 2003; 35: 171-175.

16. Kuratomi G, Iwamoto K, Bundo M, Kusumi I, Kato N, Iwata $\mathrm{N}$ et al. Aberrant DNA methylation associated with bipolar disorder identified from discordant monozygotic twins. Mol Psychiatry 2008; 13: 429-441.

17. Baranzini SE, Mudge J, van Velkinburgh JC, Khankhanian P, Khrebtukova I, Miller NA et al. Genome, epigenome and RNA sequences of monozygotic twins discordant for multiple sclerosis. Nature 2010; 464: 1351-1356

18. Fraga MF, Ballestar E, Paz MF, Ropero S, Setien F, Ballestar ML et al. Epigenetic differences arise during the lifetime of monozygotic twins. Proc Natl Acad Sci USA 2005; 102: 10604-10609.

19. Kaminsky ZA, Tang T, Wang SC, Ptak C, Oh GH, Wong AH et al. DNA methylation profiles in monozygotic and dizygotic twins. Nat Genet 2009; 41: 240-245.

20. Mill J, Dempster E, Caspi A, Williams B, Moffitt T, Craig I. Evidence for monozygotic twin (MZ) discordance in methylation level at two $\mathrm{CpG}$ sites in the promoter region of the catechol-O-methyltransferase (COMT) gene. Am J Med Genet B Neuropsychiatr Genet 2006; 141B: 421-425.

21. Petronis A, Gottesman II, Kan P, Kennedy JL, Basile VS, Paterson AD et al. Monozygotic twins exhibit numerous epigenetic differences: clues to twin discordance? Schizophr Bull 2003; 29: 169-178.

22. Rosa A, Picchioni MM, Kalidindi S, Loat CS, Knight J, Toulopoulou T et al. Differentia methylation of the X-chromosome is a possible source of discordance for bipolar disorder female monozygotic twins. Am J Med Genet B Neuropsychiatr Genet 2008; 147B: 459-462

23. Kaplan L, Foster R, Shen Y, Parry DM, McMaster ML, O'Leary MC et al. Monozygotic twins discordant for neurofibromatosis 1. Am J Med Genet A 2010; 152A: 601-606.

24. Kondo S, Schutte BC, Richardson RJ, Bjork BC, Knight AS, Watanabe $Y$ et al. Mutations in IRF6 cause Van der Woude and popliteal pterygium syndromes. Nat Genet 2002; 32: 285-289.

25. Sakuntabhai A, Ruiz-Perez V, Carter S, Jacobsen N, Burge S, Monk S et al. Mutations in ATP2A2, encoding a Ca2+ pump, cause Darier disease. Nat Genet 1999; 21: 271-277.

26. Helderman-van den Enden AT, Maaswinkel-Mooij PD, Hoogendoorn E, Willemsen $R$ Maat-Kievit JA, Losekoot $\mathrm{M}$ et al. Monozygotic twin brothers with the fragile $\mathrm{X}$ syndrome: different CGG repeats and different mental capacities. J Med Genet 1999; 36: 253-257.

27. Bruder CE, Piotrowski A, Gijsbers AA, Andersson R, Erickson S, Diaz de Stahl T et al. Phenotypically concordant and discordant monozygotic twins display different DNA copy-number-variation profiles. Am J Hum Genet 2008; 82: 763-771.

28. Kusumi I, Ohmori T, Kohsaka M, Ito M, Honma H, Koyama T. Chronobiological approach for treatment-resistant rapid cycling affective disorders. Biol Psychiatry 1995; 37: 553-559.

29. Sugawara H, Iwamoto K, Bundo M, Ueda J, Ishigooka J, Kato T. Comprehensive DNA methylation analysis of human peripheral blood leukocytes and lymphoblastoid cell lines. Epigenetics 2011; 6: 508-515.

30. Hayashi A, Kasahara T, Kametani M, Toyota T, Yoshikawa T, Kato T. Aberrant endoplasmic reticulum stress response in lymphoblastoid cells from patients with bipolar disorder. Int J Neuropsychopharmacol 2009; 12: 33-43.

31. Kato T, Ishiwata M, Mori K, Washizuka S, Tajima O, Akiyama T et al. Mechanisms of altered $\mathrm{Ca} 2+$ signalling in transformed lymphoblastoid cells from patients with bipolar disorder. Int J Neuropsychopharmacol 2003; 6: 379-389.

32. Johnson WE, Li W, Meyer CA, Gottardo R, Carroll JS, Brown M et al. Model-based analysis of tiling-arrays for ChIP-chip. Proc Natl Acad Sci USA 2006; 103: 12457-12462.

33. Li LC, Dahiya R. MethPrimer: designing primers for methylation PCRs. Bioinformatics 2002; 18: 1427-1431.

34. Kumaki Y, Oda M, Okano M. QUMA: quantification tool for methylation analysis. Nucleic Acids Res 2008; 36: W170-W175.

35. Colella S, Shen L, Baggerly KA, Issa JP, Krahe R. Sensitive and quantitative universal Pyrosequencing methylation analysis of CpG sites. Biotechniques 2003; 35: 146-150.

36. Hu XZ, Lipsky RH, Zhu G, Akhtar LA, Taubman J, Greenberg BD et al. Serotonin transporter promoter gain-of-function genotypes are linked to obsessive-compulsive disorder. Am J Hum Genet 2006; 78: 815-826.

37. Kaiser R, Muller-Oerlinghausen B, Filler D, Tremblay PB, Berghofer A, Roots I et al. Correlation between serotonin uptake in human blood platelets with the 44-bp polymorphism and the 17-bp variable number of tandem repeat of the serotonin transporter. Am J Med Genet 2002; 114: 323-328.

38. Kaiser R, Tremblay PB, Schmider J, Henneken M, Dettling M, Muller-Oerlinghausen B et al. Serotonin transporter polymorphisms: no association with response to antipsychotic treatment, but associations with the schizoparanoid and residual subtypes of schizophrenia. Mol Psychiatry 2001; 6: 179-185.

39. Hozumi N, Tonegawa S. Evidence for somatic rearrangement of immunoglobulin genes coding for variable and constant regions. Proc Natl Acad Sci USA 1976; 73: 3628-3632.

40. Oquendo MA, Hastings RS, Huang YY, Simpson N, Ogden RT, Hu XZ et al. Brain serotonin transporter binding in depressed patients with bipolar disorder using positron emission tomography. Arch Gen Psychiatry 2007; 64: 201-208.

41. Arango V, Underwood MD, Boldrini M, Tamir H, Kassir SA, Hsiung S et al. Serotonin $1 \mathrm{~A}$ receptors, serotonin transporter binding and serotonin transporter mRNA expression in the brainstem of depressed suicide victims. Neuropsychopharmacology 2001; 25: 892-903.

42. Cho HJ, Meira-Lima I, Cordeiro Q, Michelon L, Sham P, Vallada H et al. Population-based and family-based studies on the serotonin transporter gene polymorphisms and bipolar disorder: a systematic review and meta-analysis. Mol Psychiatry 2005; 10: 771-781.

43. Kunugi $H$, Hattori M, Kato $T$, Tatsumi M, Sakai T, Sasaki $T$ et al. Serotonin transporter gene polymorphisms: ethnic difference and possible association with bipolar affective disorder. Mol Psychiatry 1997; 2: 457-462.

44. Mill J, Petronis A. Molecular studies of major depressive disorder: the epigenetic perspective. Mol Psychiatry 2007; 12: 799-814.

45. Beach SR, Brody GH, Todorov AA, Gunter TD, Philibert RA. Methylation at SLC6A4 is linked to family history of child abuse: an examination of the lowa Adoptee sample. Am J Med Genet B Neuropsychiatr Genet 2010; 153B: 710-713.

46. van IMH, Caspers K, Bakermans-Kranenburg MJ, Beach SR, Philibert R. Methylation matters: interaction between methylation density and serotonin transporter genotype predicts unresolved loss or trauma. Biol Psychiatry 2010; 68: 405-407.

47. Philibert R, Madan A, Andersen A, Cadoret R, Packer H, Sandhu H. Serotonin transporter mRNA levels are associated with the methylation of an upstream $\mathrm{CpG}$ island. $\mathrm{Am} \mathrm{J} \mathrm{Med}$ Genet B Neuropsychiatr Genet 2007; 144B: 101-105.

48. Weaver IC, Cervoni N, Champagne FA, D'Alessio AC, Sharma S, Seckl JR et al. Epigenetic programming by maternal behavior. Nat Neurosci 2004; 7: 847-854.

49. Wong CC, Caspi A, Williams B, Craig IW, Houts R, Ambler A et al. A longitudinal study of epigenetic variation in twins. Epigenetics 2010; 5: 516-526.

50. Connor CM, Akbarian S. DNA methylation changes in schizophrenia and bipolar disorder. Epigenetics 2008; 3: 55-58.

51. Kato T, Iwamoto K, Kakiuchi C, Kuratomi G, Okazaki Y. Genetic or epigenetic difference causing discordance between monozygotic twins as a clue to molecular basis of mental disorders. Mol Psychiatry 2005; 10: 622-630.

(c)

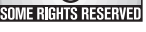
under the Creative Commons Attribution-Noncommercial -No Derivative Works 3.0 Unported License. To view a copy of this license, visit http://creativecommons.org/licenses/by-nc-nd/3.0/

\section{Supplementary Information accompanies the paper on the Translational Psychiatry website (http://www.nature.com/tp)}

